CORIGINAL ARTICLE -

Volume 14 Issue 22019

DOI: 10.21315/aos2019.14.2.377

ARTICLE INFO

Submitted: 04/04/2019

Accepted: 21/11/2019

Online: 30/12/2019

\section{Fluoride Content, Cost and Labelling of Commercially Available Toothpastes in Malaysia}

\author{
Juwairiah Wafa Anis ${ }^{a}$, Mohammad Haniff Abdul Hamida, \\ Norasmiha Mohd Nor ${ }^{b}$, Nor Azlida Mohd Nor ${ }^{c \star}$ \\ ${ }^{a}$ Faculty of Dentistry, Universiti Malaya, 50603 Kuala Lumpur, \\ Malaysia \\ ${ }^{b}$ Department of Production $\mathcal{E}$ Operations Management, Universiti Tun \\ Hussein Onn Malaysia, 86400 Batu Pahat, fohor, Malaysia \\ ${ }^{c}$ Department of Community Oral Health \& Clinical Prevention, Faculty \\ of Dentistry, Universiti Malaya, 50603 Kuala Lumpur, Malaysia \\ `Corresponding author: azlida@um.edu.my
}

To cite this article: Anis JW, Abdul Hamid MH, Mohd Nor N, Mohd Nor NA (2019). Fluoride content, cost and the labelling of commercially available toothpastes in Malaysia. Arch Orofac Sci, 14(2): 113-131. https://doi.org/10.21315/aos2019.14.2.377

To link to this article: https://doi.org/10.21315/aos2019.14.2.377

\begin{abstract}
The objectives of this study were to document the ingredients of toothpastes that are available in Malaysia and to explore the cost, manufacturers' recommendations for toothpaste use and their labelling practices. Commercially available toothpastes were identified from online and offline (over-the-counter) markets. An online search was conducted through major online shopping websites in Malaysia. For the offline market, selected major pharmacies, supermarkets, family marts and traditional medicine stores in the Klang Valley area were visited. Relevant information (i.e. the type of fluoride, fluoride concentration, type of abrasive agent, price per item and recommendation for use) on the toothpaste packaging were recorded. Data were analysed descriptively using Microsoft Excel and SPSS. A total of 176 brands of toothpaste were identified in this survey. About $74.57 \%$ and $25.43 \%$ were indicated for adults and children use respectively. Among these, $47.98 \%$ were labelled as fluoridated toothpaste and $50.29 \%$ were labelled as non-fluoridated toothpaste. Different units of labelling were used to indicate the presence of fluoride and its concentration. Generally, common abrasive agents used were silica, phosphates and carbonates. The cost of toothpaste per $10 \mathrm{~g}$ ranged from RM0.10 to RM9.50, with mean of RM1.90 (SD 1.93). Manufacturers' recommendation of toothpaste use varied with a small proportion following evidence-based recommendations. In conclusion, there were a large variety of toothpastes available in Malaysia. The cost of toothpaste varied greatly across brands. There was a lack of standardisation regarding labelling practices and recommendations of use by the manufactures, which may place the consumers and dental health professionals at a disadvantage.
\end{abstract}

Keywords: Cost; fluoride; labelling practice; toothpaste; Malaysia.

\section{INTRODUCTION}

The prevalence of dental caries has shown a decreasing trend worldwide (Frencken et al., 2017). Several factors contributed to caries reduction. One of the most significant factor contributing to this trend is the use of fluoride (Petersen and Ogawa, 2016). It has been well documented in the literature that fluoride can enhance remineralisation and retard the demineralisation process, hence preventing caries progression 
(O'Mullane et al., 2016; Petersen and Ogawa, 2016). The main mode of fluoride delivery is topical which is frequently used in toothpaste (Canadian Paediatric Society, 2002; O'Mullane et al., 2016). To ensure effective fluoride delivery using toothpaste, appropriate labelling is important to aid consumers in making the correct choices.

Although there is strong evidence on effectiveness of fluoride in caries prevention, there is still on-going controversy over fluoride use, either in drinking water or toothpaste. Resistance has been growing worldwide against fluoride, emphasising on the possible risk of toxicity (Aoun et al., 2018). Thus, information regarding the number of non-fluoride toothpastes in the market is important for practicing dental professionals to advise their patients appropriately.

Due to globalisation and advancement in technology, there has been an influx of toothpaste availability in Malaysia, either through online or over-the-counter products. Thus, purchasing the correct toothpaste may be confusing to the consumers. In developed countries such as the United States, the standard of labelling practices is strictly monitored under The Regulation of Toothpaste by Food and Drug Administration (FDA). It was also highlighted that the products may not be marketed if the labelling is misleading or the advertisement contains false claims (Sandier, 1997). In Malaysia, a guide on the use of fluoride, published by the Malaysian Dental Council, states that the fluoride concentration in toothpaste for the use of children under six years old should be displayed with a specific indication on the packaging and container (Malaysian Dental Council, 2009). However, a recent local study by Gundavarappa et al. (2017) reported poor labelling practice of toothpaste marketed in Malaysia. The problem persists, although this issue was highlighted 20 years ago by Musa and Saub (1998). This is worrying as consumers rely on the labelling to get key information about the product before purchasing.

In addition, there are several factors associated with the choice of toothpaste among consumers. These factors include the ingredient, brand, perceived performance and manufacturers' recommendation of the product (Opeodu and Gbadebo, 2017). For example, in terms of the ingredient, some consumers may have concerns about the fluoride content, while others are concerned about the herbal content. Although the importance of fluoride toothpaste has been well documented, a study has reported that fluoride-containing toothpaste may not be globally affordable (Goldman et al., 2008). The cost of the product may be one of the major factors that may influence consumer's choice of toothpaste. Whether the cost of fluoridated and non-fluoridated toothpaste differ significantly in Malaysia remains unexplored.

The previous two local studies (Musa and Saub, 1998; Gundavarappa et al., 2017) only included a small samples size and mainly focused on the type of fluoride content in the toothpaste. Details of other information on the packaging were not reported. Therefore, it is essential to know what is available in the current market in Malaysia. The findings will be beneficial for the consumers to assist them in choosing an appropriate toothpaste. Also, the data will serve as a guide for dental health professionals to provide specific advice on toothpaste for their patients. The findings will also be useful to validate research data on the self-reported questionnaire about the type of toothpaste used among the study's participants. Thus, the objectives of this study were to document the types, ingredients and the cost of toothpaste available in Malaysia and to explore manufacturers' recommendation of toothpaste used and their labelling practice. 


\section{MATERIALS AND METHODS}

This research has been approved by the Medical Ethics Committee, Faculty of Dentistry, University of Malaya [DF CO1804/0014(U)].

Toothpaste availability was identified from both online and offline (over-the-counter) markets. An online survey was conducted by searching through major online shopping websites such as Lazada, 11th Street, Shopee, Tesco, Watsons and Guardian. Keywords such as; 'toothpaste' AND/OR 'fluoridated' AND/OR 'non-fluoridated' were used during the online search. For the offline market, selected major pharmacies, supermarkets, family marts and traditional medicine stores in the Klang Valley area were visited. An assumption was made that various toothpastes available in Malaysia were available in the Klang Valley market. Identified toothpaste products from the search were recorded. The selection of the stores were based on popularity and logistic reasons. Data collection period was from 1 April to 7 August 2018.

The list of products from online and offline markets was compared, and duplicates were excluded from the sample. However, if the same brand manufactured both fluoridated and non-fluoridated products, both items were recorded. The information written on the packaging was recorded in Microsoft Excel spreadsheet; the country of manufacture, the date of expiry, the date of manufacture and price per item $(x /$ gram), halal labelling and recommendation for use. Regarding the ingredients, the information on the packaging was also recorded to identify the descriptive names of fluoride compounds, their concentrations and the types of abrasives. The data were categorised into four groups for purposes of data presentation: fluoridated toothpaste for adults, non-fluoridated toothpaste for adults, fluoridated toothpaste for children and nonfluoridated toothpaste for children. Children toothpastes were identified based on age indication written on the box or by childrenoriented packaging. Toothpastes that did not fall within the children's category were recorded as for adult use. To allow price comparison across brands, the price for $10 \mathrm{~g}$ was calculated. The cost of toothpastes were further re-coded into low ( $<R M 1)$, medium (RM1-RM4) and high cost (RM4) for data presentation and further analysed by age group and fluoride content. The data were analysed descriptively using Microsoft Excel and SPSS version 23.

\section{RESULTS}

\section{Manufacturers' Labelling Practice}

In total, 176 brands of toothpastes were identified from this market survey. Most of the toothpaste available in the online market were also available in the physical stores. Amongst these, $47.98 \%$ were labelled as fluoridated toothpaste, where as $50.29 \%$ were labelled as non-fluoridated toothpaste or had no fluoride compound in their list of ingredients. About $25.43 \%$ brands were recommended for children, and the remaining $74.57 \%$ of toothpastes were recommended for adult use. Only $1.73 \%$ of the brands manufactured both fluoridated and non-fluoridated toothpaste. The majority of the products were available in paste/gel form, while a very small proportion $(2.89 \%)$ were in powder form. The powder based products were only available for the adult category.

Regarding other labelling information, most of the toothpastes $(65.91 \%)$ labelled the expiry date on their packaging. However, only $42.05 \%$ of the toothpaste manufacturers provided the information about the manufacturing date on the packaging. Only half of the products labelled both the expiring and manufacturing dates. About $38.07 \%$ indicated halal labelling on their packaging box. Summary results of manufacturers' labelling practice is shown in Table 1. 
Most of the adult toothpastes were imported $(63.57 \%)$, and only $33.33 \%$ of the brands sold in Malaysia were manufactured locally. Among the imported toothpastes, the majority was from the United States $(14.63 \%)$, followed by China $(12.20 \%)$ and India $(10.97 \%)$. Around $3.1 \%$ did not specify the country of manufacture. Similar to adult toothpastes, most of the children toothpastes (59.09\%) were imported from other countries, and only $36.36 \%$ were locally produced. There were $4.55 \%$ brands of toothpaste that did not state its manufacturing origin. The imported toothpastes were mainly from the United States $(n=4)$, Australia $(n=4)$, Italy $(n=3)$, Thailand $(n=2)$, Taiwan $(n=2)$, Vietnam $(n=2)$ and Russia $(n=2)$.

Table 1 Summary results of manufacturers' labelling practice

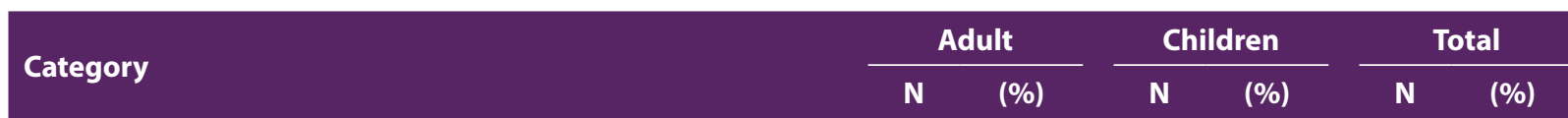

INGREDIENTS

\section{Fluoride compound} Labelling unit for fluoride
concentration

$\begin{array}{ll} & \text { Not stated } \\ & \text { Total } \\ \text { Abrasive agents (present/ } & \text { Stated } \\ \text { absent) } & \text { Abrasive free } \\ & \text { Not stated } \\ & \text { Total } \\ & \text { Silica } \\ \text { Type of common abrasive } & \text { Carbonates } \\ \text { compound } & \text { Phosphates } \\ & \text { Others } \\ & \text { Total } \\ & \text { Yes } \\ \text { Herbal or organic } & \text { No } \\ \text { ingredient } & \text { Total }\end{array}$
(NaMFP)

$\mathrm{NaF}+\mathrm{NaMFP}$

Not stated

Total

Not stated

Total
Fluoridated toothpaste

Sodium Fluoride (NaF)

Sodium Monofluorophosphate

Calcium Fluoride (CaF)

Non-fluoridated toothpaste

Labelled fluoride-free

Parts per million (ppm)

Percentage (\%), \% w/w, \% w/v*

34.85

$12 \quad 12.18$

$31 \quad 46.97$

$66 \quad 37.5$

$121 \quad 93.08$

$1 \quad 0.77$

$8 \quad 6.15$

$130 \quad 73.86$

100

$51 \quad N / A$

N/A

$34 \mathrm{~N} / \mathrm{A}$

$55 \mathrm{~N} / \mathrm{A}$

240 N/A

$55 \quad 42.31$

$75 \quad 57.69$

$130 \quad 73.86$

\begin{tabular}{|c|c|c|c|}
\hline 9 & 45.00 & 27 & 31.40 \\
\hline 8 & 40.00 & 48 & 55.81 \\
\hline 1 & 5.00 & 5 & 5.81 \\
\hline 0 & 0.00 & 1 & 1.16 \\
\hline 2 & 10.00 & 5 & 5.81 \\
\hline 20 & 11.36 & 86 & 48.86 \\
\hline 17 & 65.38 & 48 & 53.33 \\
\hline 9 & 34.62 & 42 & 46.67 \\
\hline 26 & 14.77 & 90 & 51.11 \\
\hline 6 & 30.00 & 29 & 33.72 \\
\hline 3 & 15.00 & 15 & 17.44 \\
\hline 11 & 55.00 & 42 & 48.84 \\
\hline 20 & 11.36 & 86 & 48.86 \\
\hline 42 & 91.30 & 163 & 92.61 \\
\hline 1 & 2.17 & 2 & 1.14 \\
\hline 3 & 6.52 & 11 & 6.25 \\
\hline 46 & 26.14 & 176 & 100 \\
\hline 35 & N/A & 135 & $\mathrm{~N} / \mathrm{A}$ \\
\hline 7 & $\mathrm{~N} / \mathrm{A}$ & 58 & $\mathrm{~N} / \mathrm{A}$ \\
\hline 16 & $\mathrm{~N} / \mathrm{A}$ & 50 & $\mathrm{~N} / \mathrm{A}$ \\
\hline 15 & N/A & 70 & $\mathrm{~N} / \mathrm{A}$ \\
\hline 73 & N/A & 313 & N/A \\
\hline 10 & 21.74 & 65 & 36.93 \\
\hline 36 & 78.26 & 111 & 63.07 \\
\hline 46 & 26.14 & 176 & 100 \\
\hline
\end{tabular}

(Continued on next page) 
Table 1 (Continued)

\begin{tabular}{|c|c|c|c|c|c|c|c|}
\hline \multirow{2}{*}{\multicolumn{2}{|c|}{ Category }} & \multicolumn{2}{|c|}{ Adult } & \multicolumn{2}{|c|}{ Children } & \multicolumn{2}{|c|}{ Total } \\
\hline & & $\mathbf{N}$ & $(\%)$ & $\mathbf{N}$ & $(\%)$ & $\mathbf{N}$ & (\%) \\
\hline \multicolumn{8}{|c|}{ OTHER LABELLING INFORMATION } \\
\hline \multirow{4}{*}{ Manufacturer } & Imported & 83 & 63.84 & 27 & 58.70 & 110 & 62.43 \\
\hline & Local & 43 & 33.07 & 16 & 34.78 & 59 & 34.10 \\
\hline & Not stated & 4 & 3.07 & 3 & 6.52 & 7 & 3.47 \\
\hline & Total & 130 & 73.86 & 46 & 26.14 & 176 & 100 \\
\hline \multirow[t]{3}{*}{ Manufacturing date } & Stated & 53 & 40.77 & 21 & 45.65 & 74 & 42.05 \\
\hline & Not stated & 77 & 59.23 & 25 & 54.35 & 102 & 57.95 \\
\hline & Total & 130 & 73.86 & 46 & 26.14 & 176 & 100 \\
\hline \multirow[t]{3}{*}{ Expiry date } & Stated & 94 & 72.31 & 22 & 47.83 & 116 & 65.91 \\
\hline & Not stated & 36 & 27.69 & 24 & 52.17 & 60 & 34.09 \\
\hline & Total & 130 & 73.86 & 46 & 26.14 & 176 & 100 \\
\hline \multirow[t]{3}{*}{ Halal labelling } & Stated & 54 & 41.54 & 13 & 28.26 & 67 & 38.07 \\
\hline & Not stated & 76 & 58.46 & 33 & 71.74 & 109 & 61.93 \\
\hline & Total & 130 & 73.86 & 46 & 26.14 & 176 & 100 \\
\hline
\end{tabular}

*\% w/w: percentage weight/weight, \% w/v: percentage weight/volume

F: fluoride; N/A: information not available (total \% was not calculated due to multiple abrasive agents found in each toothpaste)

\section{Adult Toothpaste}

Table 2 shows the results for adult fluorided toothpastes in the Malaysian market. About half of the toothpastes $(50.77 \%)$ contained fluoride, based on the packaging box. Different units were used to indicate the fluoride content such as percentage $(\%)$, parts per million ( $\mathrm{ppm})$, percentage weight/weight $\left(\begin{array}{ll}\% & \mathrm{w} / \mathrm{w})\end{array}\right)$ and percentage weight/volume $(\% \mathrm{w} / \mathrm{v})$. The most common fluoride compound used was sodium monofluorophosphate, NaMFP (60.61\%), followed by sodium fluoride, $\mathrm{NaF}(27.27 \%)$ and calcium fluoride, $\mathrm{CaF}_{2}$ (1.52\%). Although some manufacturers claimed that their products contained fluoride, 31 brands did not state the fluoride concentration. Apart from fluoride, abrasive agents used in the toothpastes were also noted. The most common abrasive agents were silica, calcium, carbonates and phosphates. A small proportion of the identified toothpastes had calcium-based abrasives with sodium fluoride.
The remaining $49.23 \%$ of the samples were labelled as non-fluoridated toothpaste or had no fluoride compound in their list of ingredients. Results from the analysis of adult non-fluoridated toothpastes are presented in Table 3. The most common active abrasive agents for non-fluoridated toothpastes were silica and calcium carbonates. It was also noted that non-fluoridated toothpastes were mostly marketed as herbal or organic products. Some of the organic-based ingredients included were miswak, natural mint, tea tree oil, coconut oil, olive oil, aloe vera, nutmeg and neem.

\section{Children Toothpaste}

Among the children toothpastes, $43.48 \%$ were fluoridated, and the remaining $56.52 \%$ brands were non-fluoridated. The results from the analysis of children toothpastes are presented in Tables 4 and 5 . The most common fluoride compound used in childrens toothpaste were sodium fluoride, $\mathrm{NaF}$ (45\%) and sodium 
Table 2 Results of the analyses of adult fluoride toothpaste in the Malaysian market

\begin{tabular}{|c|c|c|c|c|c|}
\hline ID* & Manufacturer & Abrasive agents & Type of F & F concentration & $\begin{array}{l}\text { Price/10 g } \\
\text { (RM) }\end{array}$ \\
\hline 1 & Spain & PP, silica TPPP & $\mathrm{NaF}$ & $\begin{array}{l}\text { NaF 0.22\% } \\
\text { (995 ppm F-) }\end{array}$ & 6.30 \\
\hline 2 & USA & Silica & $\mathrm{NaF}$ & $\begin{array}{l}\text { NaF } 0.21 \% \\
\text { (950 ppm F-) }\end{array}$ & 1.40 \\
\hline 3 & China & $\mathrm{NaHCO}_{3}$, silica & Mention F only & Not stated & 0.50 \\
\hline 4 & USA & $\mathrm{Al}(\mathrm{OH})_{3}$, silica, TPPP & NaMFP & Not stated & 2.70 \\
\hline 5 & UK & $\mathrm{NaHCO}_{3}, \mathrm{Na}_{2} \mathrm{H}_{3} \mathrm{CO}_{6}$, silica, TPPP & $\mathrm{NaF}$ & $\mathrm{NaF} 0.243 \%$ & 2.50 \\
\hline 6 & Korea & Silica, TPPP & NaMFP, NaF & Not stated & 0.80 \\
\hline 7 & Korea & DCPD, silica & NaMFP & Not stated & 1.60 \\
\hline 8 & USA & $\mathrm{CaCO}_{3}, \mathrm{CaO}_{2}, \mathrm{SiO}_{2}, \mathrm{SPP}$, silica & NaMFP & NaMFP $0.15 \%$ W/V & 4.60 \\
\hline 9 & Malaysia & $\mathrm{CaCO}_{3}, \mathrm{NaHCO}_{3}, \mathrm{Na}_{2} \mathrm{CO}_{3}$, silica, TSPP & NaMFP & Not stated & 0.20 \\
\hline 10 & Indonesia & $\mathrm{CaCO}_{3}$, silica & NaMFP, NaF & $\begin{array}{l}\text { NaMFP } 0.76 \%+ \\
\text { NaF } 0.01 \%\end{array}$ & 0.30 \\
\hline 11 & Indonesia & Silica & $\mathrm{NaF}$ & Not stated & 0.40 \\
\hline 12 & China & DCPD, TSPP & NaMFP & $\begin{array}{l}\text { NaMFP } 1.1 \% \text { W/W } \\
(1,450 \text { ppm F-) }\end{array}$ & 0.40 \\
\hline 13 & China & PSTP, silica, TPPP & $\mathrm{NaF}$ & Not stated & 1.20 \\
\hline 14 & Malaysia & $\mathrm{CaCO}_{3}$, silica & NaMFP & NaMFP $0.76 \%$ W/W & 0.30 \\
\hline 15 & USA & Mica, SHMP, silica, TSP & $\mathrm{NaF}$ & $\begin{array}{l}\mathrm{NaF} 0.243 \% \\
(0.16 \% \text { W/V F-) }\end{array}$ & 5.00 \\
\hline 16 & Switzerland & CA, DSP, silica & $\mathrm{NaF}$ & $\mathrm{NaF} 1,450$ ppm F- & 4.00 \\
\hline 17 & China & Mica, PSTP, silica, TSPP & $\mathrm{NaF}$ & Not stated & 0.80 \\
\hline 18 & China & DCPD, DCP, TSPP & NaMFP & Not stated & 0.60 \\
\hline 19 & India & $\mathrm{CaCO}_{3}$, silica, TSPP & NaMFP & $\begin{array}{l}\text { NaMFP } 0.76 \% \text { W/W } \\
(1,000 \text { ppm })\end{array}$ & 1.15 \\
\hline 20 & Spain & CGP, SAP, silica & NaMFP & NaMFP 1,460 ppm F- & 2.60 \\
\hline 21 & Dubai & Silica & Not stated & Not stated & 1.20 \\
\hline 22 & Malaysia & DCP, silica & NaMFP & Not stated & 0.20 \\
\hline 23 & Thailand & DSP, SHMP, silica & $\mathrm{NaMFP}, \mathrm{NaF}$ & Not stated & 0.80 \\
\hline 24 & China & $\mathrm{CaCO}_{3}$, silica & NaMFP & NaMFP $0.7 \%$ & 0.40 \\
\hline 25 & Malaysia & $\mathrm{CaCO}_{3}$, silica & NaMFP & Not stated & 0.20 \\
\hline 26 & Indonesia & PSTP, silica & NaMFP & Not stated & 0.30 \\
\hline 27 & China & $\mathrm{CaCO}_{3}$, silica & $\mathrm{NaMFP}, \mathrm{NaF}$ & $\begin{array}{l}\text { NaMFP, NaF } \\
(1,000 \text { ppm F- })\end{array}$ & 0.30 \\
\hline 28 & UK & None & $\mathrm{NaF}$ & Not stated & 1.60 \\
\hline 29 & Malaysia & DCPD, TSPP & NaMFP & Not stated & 1.35 \\
\hline 30 & Spain & Silica & $\mathrm{NaF}$ & $\mathrm{NaF} 1,450$ ppm F- & 4.90 \\
\hline 31 & India & CA, silica & $\mathrm{CaF}$ & CaF 500 ppm F- & 0.40 \\
\hline 32 & Malaysia & $\mathrm{CaCO}_{3}$, silica & NaMFP & Not stated & 0.30 \\
\hline 33 & Malaysia & $\mathrm{CaCO}_{3}$, silica & NaMFP & Not stated & 0.30 \\
\hline
\end{tabular}


Table 2 (Continued)

\begin{tabular}{|c|c|c|c|c|c|}
\hline ID* & Manufacturer & Abrasive agents & Type of F & F concentration & $\begin{array}{c}\text { Price/10 g } \\
\text { (RM) }\end{array}$ \\
\hline 34 & Italy & Silica, TPPP & NaMFP & $\begin{array}{l}\text { NaMFP } 0.72 \% \\
(950 \text { ppm F-) }\end{array}$ & 1.90 \\
\hline 35 & Myanmar & $\mathrm{CaCO}_{3}, \mathrm{MAS}, \mathrm{NaHCO} 3$, silica & NaMFP & Not stated & 0.50 \\
\hline 36 & Taiwan & $\begin{array}{l}\text { Cleansing formula, Lizey Plus- X-vc } \\
\text { whitening factor, Lizey SmoCure - } \\
\text { X-vc teeth whitening factor }\end{array}$ & NaMFP & Not stated & 6.70 \\
\hline 37 & Pakistan & None & NaMFP & NaMFP 1,400 ppm F- & 1.40 \\
\hline 38 & USA & Silica, TPPP, TSPP & $\mathrm{NaF}$ & $\mathrm{NaF} 0.234 \% \mathrm{~W} / \mathrm{W}$ & 1.55 \\
\hline 39 & Malaysia & DCP & NaMFP & Not stated & 0.50 \\
\hline 40 & India & $\mathrm{CaCO}_{3}$, SDHP, silica & NaMFP & NaMFP 100 ppm F- & 0.40 \\
\hline 41 & Malaysia & DCPD, silica, TSPP & NaMFP & Not stated & 1.40 \\
\hline 42 & UK & $\begin{array}{l}\text { DCP, glucose oxidase, silica, } \\
\text { lactoperoxidase, lysozymes }\end{array}$ & NaMFP & $\begin{array}{l}\text { NaMFP } 0.76 \% \text { W/W } \\
(1,000 \text { ppm F-) }\end{array}$ & 3.20 \\
\hline 43 & Germany & Silica, sodium citrate, ZC & $\mathrm{NaF}$ & $\mathrm{NaF} 1,450$ ppm F- & 1.30 \\
\hline 44 & Thailand & $\mathrm{NaHCO}_{3}$ & $\mathrm{NaF}$ & $\begin{array}{l}\mathrm{NaF} 0.221 \% \mathrm{~W} / \mathrm{W} \\
(1,000 \mathrm{ppm} \mathrm{F-})\end{array}$ & 1.20 \\
\hline 45 & India & None & Mention F only & F content $\sim 924$ ppm & 0.45 \\
\hline 46 & India & $\mathrm{CaCO}_{3}$ & Mention F only & F content $\sim 924$ ppm & 0.90 \\
\hline 47 & Singapore & Alumina, $\mathrm{CaCO}_{3}, \mathrm{NaHCO}_{3}$, silica & NaMFP & $\begin{array}{l}\text { NaMFP } 0.80 \% \text { W/W } \\
(1,050 \text { ppm F-) }\end{array}$ & 1.55 \\
\hline 48 & Australia & CPP, DCP, silica, SP, TSP & NaMFP & NaMFP $0.76 \%$ W/W & 2.80 \\
\hline 49 & Indonesia & $\mathrm{CaCO}_{3}$, silica & NaMFP & Not stated & 0.20 \\
\hline 50 & Spain & Silica, TPPP & $\mathrm{NaF}$ & $\mathrm{NaF} 0.33 \% \mathrm{~W} / \mathrm{W}$ & 1.65 \\
\hline 51 & Malaysia & $\mathrm{CaCO}_{3}$, tetrasodium EDTA, TSPP & NaMFP & Not stated & 0.40 \\
\hline 52 & Thailand & Silica & $\mathrm{NaF}$ & $\begin{array}{l}\mathrm{NaF} 0.221 \% \mathrm{~W} / \mathrm{W} \\
(1,000 \mathrm{ppm})\end{array}$ & 0.90 \\
\hline 53 & USA & PSTP, silica & NaMFP & Not stated & 1.90 \\
\hline 54 & Malaysia & CP, DCPD, Silica, TSPP & NaMFP & Not stated & 0.20 \\
\hline 55 & Malaysia & $\mathrm{CaCO}_{3}$, silica & NaMFP & Not stated & 0.40 \\
\hline 56 & China & CSPS, silica & $\mathrm{NaF}$ & $\begin{array}{l}\mathrm{NaF} 0.3152 \% \mathrm{~W} / \mathrm{W} \\
(1,450 \mathrm{ppm} \mathrm{F}-)\end{array}$ & 1.40 \\
\hline 57 & Vietnam & $\mathrm{CaCO}_{3}$, potassium citrate, silica & NaMFP & NaMFP 1,450 ppm F- & 0.40 \\
\hline 58 & Indonesia & $\mathrm{CaCO}_{3}$ & NaMFP & Not stated & $\mathrm{n} / \mathrm{a}$ \\
\hline 59 & Indonesia & Not stated & NaMFP & Not stated & $\mathrm{n} / \mathrm{a}$ \\
\hline 60 & Japan & silica & $\mathrm{NaF}$ & Not stated & 1.10 \\
\hline 61 & Malaysia & Not stated & NaMFP & NaMFP $0.76 \%$ & 1.20 \\
\hline 62 & Malaysia & $\mathrm{CaCO}_{3}$, pyrophosphate, silica & NaMFP & Not stated & 0.20 \\
\hline 63 & Malaysia & $\mathrm{CaCO}_{3}$, pyrophosphate, silica & NaMFP & Not stated & 0.10 \\
\hline 64 & USA & Silica, TPPP, TSPP & $\mathrm{NaF}$ & $\mathrm{NaF} 0.24 \%$ & 4.20 \\
\hline 65 & Japan & CHP, silica & $\mathrm{NaF}$ & Not stated & 8.75 \\
\hline
\end{tabular}


Table 2 (Continued)

\begin{tabular}{|c|c|c|c|c|c|}
\hline ID* & Manufacturer & Abrasive agents & Type of $F$ & F concentration & $\begin{array}{l}\text { Price/10 g } \\
\text { (RM) }\end{array}$ \\
\hline 66 & Malaysia & $\mathrm{CaCO}_{3}, \mathrm{TSPP}$ & NaMFP & NaMFP $0.76 \%$ & 0.50 \\
\hline 67 & Australia & $\mathrm{CaCO}_{3}$, silica & NaMFP & $\begin{array}{l}\text { NaMFP } 0.76 \% \text { W/W } \\
(1,000 \text { ppm F-) }\end{array}$ & 1.80 \\
\hline 68 & Malaysia & $\mathrm{CaCO}_{3}$, silica & NaMFP & Not stated & 0.70 \\
\hline 69 & Spain & PP, silica, TPPP & $\mathrm{NaF}$ & $\mathrm{NaF}$ 1,450 ppm F- & 6.70 \\
\hline 70 & Malaysia & $\mathrm{CaCO}_{3}, \mathrm{TSPP}$ & NaMFP & Not stated & 0.30 \\
\hline 71 & Japan & Alumina, $\mathrm{CaCO}_{3}$, silica & NaMFP & NaMFP 1,000 ppm F- & 0.50 \\
\hline
\end{tabular}

*Product ID: The list of all toothpaste brands is presented in Appendix 1.

F: fluoride

Type of fluoride: NaMFP, sodium monofluorophosphate; $\mathrm{NaF}$, sodium fluoride; $\mathrm{CaF}_{2}$, calcium fluoride.

Abrasive agent: $\mathrm{CaCO}_{3}$, calcium carbonate; $\mathrm{PP}$, potassium pyrophosphate; TPPP, tetrapotassium pyrphosphate; $\mathrm{NaHCO}_{3}$, sodium bicarbonate; $\mathrm{Al}(\mathrm{OH})_{3}$, Áluminium hydroxide; $\mathrm{Na}_{2} \mathrm{H}_{3} \mathrm{CO}_{6}$, sodium percarbonate; DCPD, dicalcium phosphate dihydrate; CaO ${ }_{2}$, calcium peroxide; $\mathrm{SiO}_{2}$, silicon dioxide; SPP, sodium pyrphophate; TSPP, tetrasodium pyrophosphate; PSTP, pentasodium triphosphate; SHMP, sodium hexametaphosphate; TSP, trisodium phosphate; CA, citric acid; DSP, disodium phosphate; DCP, dicalcium phosphate; CGP, calcium glycerophosphate; SAP, sodium ascorbyl phosphate; MAS, magnesium aluminium silicate; SDHP, sodium dihydrogen phosphate; CPP, calcium pyrophosphate; SP, sodium phosphate; CP, calcium phosphate; CSPS, calcium sodium phosphosilicate; CHP, calcium hydrogen phosphate; ZC, zinc citrate.

Table 3 Results of the analyses of adult non-fluoridated toothpastes in the Malaysian market

\begin{tabular}{|c|c|c|c|c|c|c|c|}
\hline ID* & Manufacturer & Abrasive Agents & $\begin{array}{l}\text { Price/ } 10 \mathrm{~g} \\
\text { (RM) }\end{array}$ & ID* & Manufacturer & Abrasive Agents & $\begin{array}{l}\text { Price/ } 10 \mathrm{~g} \\
\text { (RM) }\end{array}$ \\
\hline 72 & Not stated & Not stated & 4.10 & 91 & India & Silica & 0.40 \\
\hline 73 & Not stated & $\begin{array}{l}\mathrm{MPPCaCO} \\
\mathrm{NaHCO}_{3} \text {, silica }\end{array}$ & 0.55 & 92 & USA & $\mathrm{CaCO}_{3}$, silica & 3.00 \\
\hline 74 & Malaysia & Silica & 0.70 & 93 & Malaysia & $\mathrm{CaCO}_{3}$, silica & 0.40 \\
\hline 75 & India & Silica & 0.80 & 94 & Malaysia & DCP & 1.30 \\
\hline 76 & Thailand & Silica & 2.60 & 95 & USA & Silica & 3.80 \\
\hline 77 & China & Mica, silica & 1.00 & 96 & Malaysia & Silica & 1.10 \\
\hline 78 & Italy & Silica, TPPP & 3.35 & 97 & Australia & $\mathrm{CaCO}_{3}, \mathrm{DCPD}$, silica & 1.10 \\
\hline 79 & Malaysia & Silica & 1.00 & 98 & Malaysia & Silica & 1.50 \\
\hline $80^{\wedge}$ & Not stated & $\begin{array}{l}\mathrm{CaCO}_{3} \text {, charcoal } \\
\text { powder, } \mathrm{PP} \text {, silica, ZC }\end{array}$ & 1.40 & 99 & Malaysia & Silica & 0.30 \\
\hline 81 & Malaysia & DCP & 1.80 & 100 & Malaysia & $\mathrm{CaCO}_{3}$, silica & 0.25 \\
\hline $82^{\wedge}$ & Malaysia & Alum & 0.50 & 101 & Malaysia & $\mathrm{CaCO}_{3}$, silica & 0.40 \\
\hline 83 & China & DCP, TSPP & 0.20 & $102^{\wedge}$ & India & None & 1.70 \\
\hline 84 & France & Silica & 1.80 & 103 & India & Silica & 0.60 \\
\hline 85 & UAE & $\mathrm{CaCO}_{3}$, silica & 0.50 & 104 & Malaysia & DCP, silica, TSPP & 0.75 \\
\hline 86 & UAE & $\mathrm{CaCO}_{3}$, silica & 0.55 & 105 & India & $\mathrm{CaCO}_{3}$, silica & 0.40 \\
\hline 87 & Thailand & $\begin{array}{l}\mathrm{CaCO}_{3}, \mathrm{MAS}, \mathrm{NaHCO}_{3}, \\
\text { silica, ZC }\end{array}$ & 3.00 & 106 & Malaysia & Silica, TSP & 2.50 \\
\hline 88 & Malaysia & DCP, silica & 0.80 & 107 & Germany & $\mathrm{CaCO}_{3}$, silica & 2.50 \\
\hline 89 & Thailand & None & 1.60 & 108 & New Zealand & $\mathrm{CaCO}_{3}$ & 2.20 \\
\hline 90 & India & Silica & 0.40 & 109 & New Zealand & $\mathrm{CaCO}_{3}, \mathrm{MAS}$, silica & 3.00 \\
\hline
\end{tabular}


Table 3 (Continued)

\begin{tabular}{|c|c|c|c|c|c|c|c|}
\hline ID* & Manufacturer & Abrasive Agents & $\begin{array}{l}\text { Price/ } 10 \mathrm{~g} \\
\text { (RM) }\end{array}$ & ID* & Manufacturer & Abrasive Agents & $\begin{array}{c}\text { Price/ } 10 \mathrm{~g} \\
\text { (RM) }\end{array}$ \\
\hline 110 & Malaysia & $\mathrm{CaCO}_{3}$, silica, TSP & 2.50 & 128 & New Zealand & $\mathrm{CaCO}_{3}, \mathrm{MAS}$, silica & 1.90 \\
\hline 111 & Italy & Silica, SC & 6.40 & 129 & Malaysia & $\mathrm{CaCO}_{3}, \mathrm{TSPP}$ & 0.80 \\
\hline 112 & China & $\mathrm{CaCO}_{3}$, silica & 0.90 & 130 & Malaysia & DCP, silica, TSPP & 2.60 \\
\hline 113 & UAE & $\mathrm{CaCO}_{3}$, silica & 0.30 & 131 & Japan & $\mathrm{CaCO}_{3}$, silica & 1.75 \\
\hline 114 & Malaysia & Silica & 0.50 & 132 & Russia & Mica, silica & 2.70 \\
\hline 115 & Malaysia & $\begin{array}{l}\mathrm{CaCO}_{3}, \mathrm{CP}, \mathrm{DCPD}, \\
\text { silica }\end{array}$ & 0.30 & 133 & Malaysia & $\mathrm{CaCO}_{3}$, silica & 2.10 \\
\hline 116 & Malaysia & $\mathrm{CaCO}_{3}$, silica & 0.45 & 134 & Malaysia & $\mathrm{CaCO}_{3}$ & 0.30 \\
\hline 117 & Italy & PC, silica, ZC & 2.50 & 135 & Malaysia & DCPD, silica & 1.60 \\
\hline 118 & China & $\begin{array}{l}\text { Bamboo charcoal, } \\
\text { silica }\end{array}$ & 0.80 & 136 & Malaysia & None & 9.30 \\
\hline 119 & Malaysia & $\mathrm{CaCO}_{3}$, silica & 0.30 & $137 \wedge$ & Malaysia & None & $\mathrm{n} / \mathrm{a}$ \\
\hline 120 & Malaysia & DCPD, silica, TSPP & 1.40 & 138 & Malaysia & DCPD, silica & 2.10 \\
\hline 121 & Not stated & DCPD, silica, TSPP & 1.30 & 139 & India & $\mathrm{CaCO}_{3}$ & 0.75 \\
\hline 122 & Malaysia & DCPD, silica & 0.90 & $140^{\wedge}$ & India & None & 1.00 \\
\hline 123 & USA & $\mathrm{CaCO}_{3}$, silica & 2.50 & 141 & Malaysia & $\mathrm{CaCO}_{3}$, silica, TSPP & 1.50 \\
\hline 124 & Korea & Silica & 2.30 & 142 & Taiwan & Activated carbon, CP & 1.80 \\
\hline 125 & Malaysia & $\begin{array}{l}\mathrm{CaCO}_{3}, \mathrm{DCPD} \text {, silica, } \\
\text { TSPP }\end{array}$ & 0.65 & 143 & USA & $\mathrm{NaHCO}_{3}$, silica & 1.40 \\
\hline 126 & Malaysia & $\mathrm{DCP}, \mathrm{TSPP}$ & 0.30 & 144 & USA & $\mathrm{CaCO}_{3}, \mathrm{NaHCO}_{3}$ & 4.10 \\
\hline 127 & Thailand & $\mathrm{CaCO}_{3}$ & 2.40 & & & & \\
\hline
\end{tabular}

*Product ID: The list of all toothpaste brands is presented in Appendix 1.

$\wedge$ Powder-based product

Abrasive agents: $\mathrm{CaCO}$ calcium carbonate; $\mathrm{PP}$, potassium pyrophosphate; TPPP, tetrapotassium pyrophosphate; $\mathrm{NaHCO}_{3}$, sodium bicarbonate; DCPD, dicalcium phosphate dihydrate; TSPP, tetrasodium pyrophosphate; TSP, trisodium phosphate; DCP, dicalcium phosphate; $\mathrm{MAS}$, magnesium aluminium silicate; $\mathrm{CP}$, calcium phosphate; $\mathrm{ZC}$, zinc citrate; $\mathrm{SC}$, sodium citrate; $\mathrm{PC}$, potassium citrate.

monofluorophosphate, NaMFP (40\%) as presented in Table 4. Only one brand (5\%) claimed to have included both $\mathrm{NaF}$ and NaMFP in the ingredients. Similar to adults toothpastes, there was a lack of standardisation regarding labelling fluoride concentration units on the display packaging such as using percentage (\%), parts per million (ppm), percentage weight/weight $(\% \mathrm{w} / \mathrm{w})$ and percentage weight/volume $(\%$ $\mathrm{w} / \mathrm{v})$.

Abrasive agents were also used in the children toothpastes. Compounds of silica were identified as the most commonly used agent in the ingredients, followed by compounds of phosphates and carbonates.
There was one toothpaste that claimed to be of purely organic ingredients and contained no abrasive agents. Furthermore, three brands of toothpastes did not mention any abrasive agents on their packaging.

\section{Cost of Toothpaste}

Toothpastes were marketed at different weights, and the cost varied across brands. The adult toothpastes were marketed with product weights ranged from $25 \mathrm{~g}$ to $250 \mathrm{~g}$ while children toothpastes ranged from $19 \mathrm{~g}$ to $114 \mathrm{~g}$. To allow consistency of reporting and comparing across different brands, the price for each product was recalculated at $10 \mathrm{~g}$. The cost of toothpaste varied greatly 


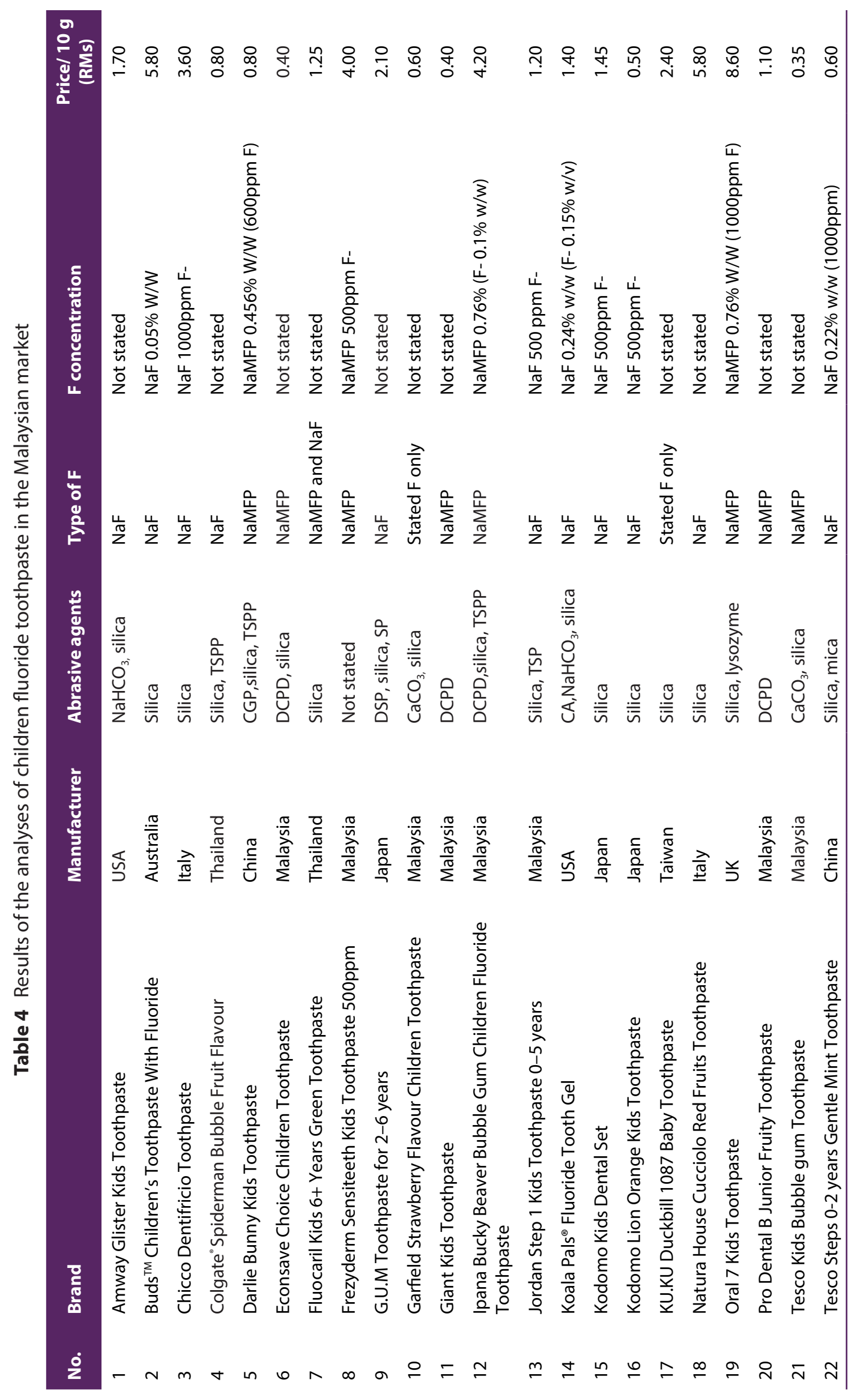


across brands ranging from low, medium to high cost. In general, the price of toothpastes per $10 \mathrm{~g}$ ranged from RM0.10 to RM9.50 with mean of RM1.90 (S.D. 1.93). When looking into toothpastes with fluoride content, the price of fluoridated toothpastes were lower than the non-fluoridated toothpastes as shown in Table 6. Broadly similar pattern was observed for both children and adult toothpaste.

Table 5 Results of the analyses of children non-fluoridated toothpaste in the Malaysian market

\begin{tabular}{|c|c|c|c|c|}
\hline No. & Brand & Manufacturer & Abrasive Agents & $\begin{array}{l}\text { Price/ } 10 \mathrm{~g} \\
\text { (RM) }\end{array}$ \\
\hline 1 & Aquafresh First Teeth Baby Toothpaste & USA & Silica, SC & 7.80 \\
\hline 2 & Coslys Junior Organic Toothpaste & France & CA, mica, silica & 1.60 \\
\hline 3 & Fiffy Kids Toothpaste & Malaysia & $\mathrm{CaCO}_{3}, \mathrm{DCPD}$, silica, TSDP & 0.70 \\
\hline 4 & $\begin{array}{l}\text { FrezyDerm SensiTeeth First Toothpaste 6+ } \\
\text { months }\end{array}$ & Not stated & Not stated & 5.00 \\
\hline 5 & Grants Kid Natural Toothpaste & Australia & $\mathrm{CaCO}_{3}, \mathrm{DCPD}$, silica & 2.30 \\
\hline 6 & Guardian Infant Oral Set & Malaysia & Silica & 3.70 \\
\hline 7 & Hito Children's Toothpaste with Xylitol & Not stated & Mica, silica & 4.60 \\
\hline 8 & $\begin{array}{l}\text { Intelligent Children Toothpaste with Natural } \\
\text { Enzymes }\end{array}$ & Taiwan & Lactoperoxidase, lysozyme & 4.70 \\
\hline 9 & Jack N' Jill Natural Toothpaste & Australia & Silica & 6.00 \\
\hline 10 & Kaydean Natural Toothpaste & Malaysia & Not stated & 4.50 \\
\hline 11 & LeRoselle Kids Toothpaste & Malaysia & DCP, silica, TSPP & 0.90 \\
\hline 12 & $\begin{array}{l}\text { Little Innoscents Milky Whites } \\
\text { OrganicToothpaste }\end{array}$ & Australia & $\mathrm{CaCO}_{3}, \mathrm{NaHCO}_{3}$ & 3.80 \\
\hline 13 & Logodent Zahngel Spearmint Kids Toothpaste & Germany & $\mathrm{NaHCO}_{3}$, silica & 6.90 \\
\hline 14 & Morning Kiss Toothpaste for Kids 3-12 Years & Malaysia & CGP, silica, SMS & 0.75 \\
\hline 15 & Mu'min Junior Toothpaste & Malaysia & DCPD, silica, TSPP & 0.40 \\
\hline 16 & NeBiolinaBebé First Teeth Toothpaste & Italy & Silica & 5.50 \\
\hline 17 & Oral 7 for Tiny Teeth Gel & Ireland & Lactoperoxidase, silica & 9.50 \\
\hline 18 & Patanjali Dant Kanti Junior Toothpaste & India & None & 0.40 \\
\hline 19 & Perioe Kids Toothpaste & Korea & Silica & 1.50 \\
\hline 20 & Pigeon Children Toothpaste & Indonesia & DCPD, TMP & 1.60 \\
\hline 21 & Pureen Kids Toothpaste & Malaysia & CPP, DCPD, silica, TSPP & 0.70 \\
\hline 22 & R.O.C.S Baby Toothpaste Mild Care & Russia & DCPD, silica & 4.70 \\
\hline 23 & Raiya Junior Children Toothpaste & Malaysia & DCPD: & 0.55 \\
\hline 24 & $\begin{array}{l}\text { Splat Junior Natural Toothpaste for Kids 2-6 } \\
\text { Years }\end{array}$ & Russia & Lactoperoxidase, silica & 2.70 \\
\hline 25 & $\begin{array}{l}\text { Splat Junior Natural Toothpaste for Kids 6-11 } \\
\text { Years }\end{array}$ & Russia & Lactoperoxidase, mica, silica & 2.70 \\
\hline 26 & Xylin Toothpaste for Kids & Malaysia & $\mathrm{CaCO}_{3}$, silica, TSPP & 1.20 \\
\hline 27 & Young Living KidScents Slique Toothpaste & USA & $\mathrm{CaCO}_{3}, \mathrm{NaHCO}_{3}$ & 2.90 \\
\hline
\end{tabular}

Abrasive agents: $\mathrm{CaCO}$ calcium carbonate; $\mathrm{NaHCO}_{3}$, sodium bicarbonate; DCPD, dicalcium phosphate dihydrate; TSPP, tetrasodium pyrophosphate; CA, citric acid; DCP, dicalcium phosphate; CGP, calcium glycerophosphate; $\mathrm{CPP}$, calcium pyrophosphate; PP, pyrophosphate; SC, sodium citrate; TSDP, tetrasodium diphosphate; SMS, sodium magnesium silicate; TMP, trimagnesium phosphate. 
Table 6 The cost of toothpaste by age group and fluoride content

\begin{tabular}{|c|c|c|c|c|c|c|}
\hline \multirow[b]{2}{*}{ Category } & \multicolumn{3}{|c|}{ Fluoridated } & \multicolumn{3}{|c|}{ Non-fluoridated } \\
\hline & $\begin{array}{c}\text { Adults } \\
\text { N (\%) }\end{array}$ & $\begin{array}{c}\text { Children } \\
\text { N (\%) }\end{array}$ & $\begin{array}{l}\text { Total } \\
\text { N (\%) }\end{array}$ & $\begin{array}{c}\text { Adults } \\
\text { N (\%) }\end{array}$ & $\begin{array}{c}\text { Children } \\
\text { N (\%) }\end{array}$ & $\begin{array}{l}\text { Total } \\
\text { N (\%) }\end{array}$ \\
\hline$<$ RM1.00 (low) & $35(50.7)$ & $8(36.4)$ & $43(47.3)$ & $33(45.8)$ & $7(25.9)$ & $40(40.4)$ \\
\hline RM1.00-RM4.00 (med) & $26(37.7)$ & $10(45.5)$ & $36(39.6)$ & $35(48.6)$ & $10(37.0)$ & $45(45.4)$ \\
\hline > RM4.00 (high) & $8(11.6)$ & $4(18.2)$ & $12(13.2)$ & $4(5.6)$ & $10(37.1)$ & $14(14.1)$ \\
\hline
\end{tabular}

\section{Manufacturers' Recommendation for Toothpaste Use}

The majority of manufacturers provided prevention advice such as toothbrushing frequency, amount of toothpaste to be used, post-brushing advice, brushing technique, dietary sugar advice and frequency of dental visits. Table 7 summarises the manufacturers' instruction of toothpaste use based on their packaging label.

Some manufacturers provided recommendation on toothpaste use. For example, brushing twice daily (36.36\%), pea-sized amount of toothpaste for children $(36.36 \%)$ and adult supervision when children are brushing (14.20\%). Some brands recommended brushing after meals. Variation of messages was also observed regarding the amount of toothpaste to be used and post-brushing advice. For example, $11.36 \%$ brands suggested that the amount of toothpaste for adults was equal to the full toothbrush bristle length, and only $1.7 \%$ brands recommend that adults use a peasized amount. Regarding post-brushing advice, about $40.77 \%$ adults toothpaste brands and $63.04 \%$ children toothpaste brands incorporated instructions to spit-out

Table 7 Manufacturers' recommendation of toothpaste use

\begin{tabular}{|c|c|c|c|c|c|c|}
\hline & \multirow[b]{2}{*}{ Description } & \multicolumn{2}{|c|}{ Adults } & \multicolumn{2}{|c|}{ Children } & \multirow{2}{*}{$\begin{array}{l}\text { Total } \\
\text { N (\%) }\end{array}$} \\
\hline & & $\begin{array}{c}F \\
N(\%)\end{array}$ & $\begin{array}{l}\text { NF } \\
\text { N (\%) }\end{array}$ & $\begin{array}{c}\mathbf{F} \\
\mathbf{N}(\%)\end{array}$ & $\begin{array}{l}\text { NF } \\
\text { N (\%) }\end{array}$ & \\
\hline \multirow{4}{*}{$\begin{array}{l}\text { Frequency of } \\
\text { toothbrushing }\end{array}$} & Twice $(2 x)$ & $30(23.08)$ & $13(10.00)$ & $8(17.39)$ & $13(28.26)$ & $64(36.36)$ \\
\hline & 3 times $(3 x)$ & $7(5.38)$ & 0 & $1(2.17)$ & 0 & $8(4.55 \%)$ \\
\hline & $>3 x$ or after meals & $1(0.77)$ & $8(6.15)$ & $1(2.17)$ & $1(2.17)$ & $11(6.25)$ \\
\hline & $\begin{array}{l}\text { Combined (brush at least } 2 \mathrm{x} \\
\text { and brush after meals) }\end{array}$ & $12(9.23)$ & $8(6.15)$ & $4(8.70)$ & 0 & $24(13.64)$ \\
\hline \multirow{5}{*}{$\begin{array}{l}\text { Amount of } \\
\text { toothpaste }\end{array}$} & Whole bristle length & $16(12.31)$ & $4(3.08)$ & 0 & 0 & $20(11.36)$ \\
\hline & Pea-sized (children) & $35(26.9)$ & $8(6.15)$ & $13(28.26)$ & $8(17.39)$ & $64(36.36)$ \\
\hline & Pea-sized (adult) & $0(0.00)$ & $3(2.31)$ & N/A & 0 & $3(1.70)$ \\
\hline & Small amount & $0(0.00)$ & $1(0.77)$ & $2(4.35)$ & $6(13.04)$ & $9(5.11)$ \\
\hline & Others & $3^{\mathrm{a}}(2.31)$ & 0 & $1^{\mathrm{b}}(2.17)$ & $6^{c}(13.04)$ & $10(5.68)$ \\
\hline \multirow{3}{*}{$\begin{array}{l}\text { Post-brushing } \\
\text { advice }\end{array}$} & Rinse & $21(16.15)$ & $12(9.23)$ & $11(23.91)$ & $11(23.91)$ & $55(31.25)$ \\
\hline & Rinse thoroughly & $2(1.54)$ & $3(2.31)$ & $1(2.17)$ & 0 & $6(3.41)$ \\
\hline & Do not swallow/spit out & $40(30.77)$ & $13(10.00)$ & 18 (39.13) & $11(23.91)$ & $82(46.59)$ \\
\hline \multicolumn{2}{|c|}{ Supervised toothbrushing } & \multicolumn{2}{|c|}{ Not relevant } & $15(32.61)$ & $10(21.74)$ & $25(14.20)$ \\
\hline \multicolumn{2}{|c|}{$\begin{array}{l}\text { Not stated (brand without any recommendation/ } \\
\text { guideline of toothpaste use) }\end{array}$} & $8(6.15)$ & $24(18.46)$ & $5(10.87)$ & 0 & $37(21.02)$ \\
\hline
\end{tabular}

${ }^{a} 1 \mathrm{~cm}(n=2), 2.5 \mathrm{~cm}(n=1)$ of toothpaste, ${ }^{b} 1 \mathrm{~cm}$ of toothpaste, ${ }^{\mathrm{c}}$ small amount $(n=6)$ of toothpaste.

$\mathrm{F}$, fluoridated; $\mathrm{NF}$, non-fluoridated toothpaste. 
after use and not to swallow the toothpaste. However, there were adult toothpaste $(25.38 \%)$ and children toothpaste $(47.82 \%)$ brands that incorporated instruction to rinse after brushing.

Of all the identified toothpastes in the present study, there were brands of adult toothpaste $(24.61 \%)$ and brands of children toothpaste $(10.87 \%)$ that did not specify any guidelines of its use.

\section{DISCUSSION}

In general, about half of the toothpastes $(47.98 \%)$ identified in this survey were fluoridated, and the remaining toothpastes were non-fluoridated (50.29\%). The findings indicate the growing number of non-fluoridated toothpastes in the Malaysian market (Musa and Saub, 1998). Therefore, continuous efforts to improve public awareness on the use of fluoridated toothpaste is crucial. This is particularly important in states without fluoridated water such as Kelantan and areas that have ceased providing fluoridated water such as Pahang (in 2012) (Ministry of Health Malaysia, 2018). Hence, the population approach to caries prevention in these areas mainly rely on fluoridated toothpaste (O'Mullane et al., 2016; Petersen and Ogawa, 2016).

There was a lack of standardisation regarding labelling practices among manufacturers. For example, about $48.84 \%$ did not indicate the concentration of fluoride contained in the paste. Of those with fluoride content, some provided information of fluoride concentration in $\% \mathrm{w} / \mathrm{w}, \% \mathrm{w} / \mathrm{v}$ instead of ppm. Similar findings have been reported by previous local studies (Musa and Saub, 1998; Gundavarappa et al., 2017). Poor labelling practices may confuse the consumers to make an informed choice of toothpaste. In addition, dental professionals may also be unaware of the concentration of fluoride present in the toothpastes when presented in different units as many evidence-based recommendations use the ppm unit. On the basis of the available information, it may also cause difficulty for a dentists to make specific recommendations to patients.

There was a wide range of toothpastes with different fluoride concentrations available in the local market. For example, adult toothpastes had fluoride concentrations ranging from 500 to $1,460 \mathrm{ppm}$, while children toothpastes had fluoride ranging from 500 to $1,000 \mathrm{ppm}$. Also, there were a number of products with low fluoride concentration $(<500 \quad \mathrm{ppm})$. Evidence suggests the use of fluoridated toothpaste with a minimum concentration of $1,000 \mathrm{ppm}$ is effective in caries prevention (Marinho et al., 2003). However, there is still variation in the recommended fluoride concentration in different countries, particularly for children. Many developed countries like the United Kingdom and New Zealand no longer recommend the use of low fluoride toothpaste at 500-600 ppm for children (Ministry of Health, New Zealand, 2009; Public Health England, 2017). In contrast, Australian guidelines recommend the use of low fluoride toothpaste (400-550 ppm) for children aged 18 months to 6 years (Spencer, 2006).

Apart from fluoride concentration, the quantity of toothpaste that should be used has been heavily debated in the literature. The use of a smear ( $\leq 3$ years) and peasized amount ( $>3$ years) of toothpaste are recommended based on the Malaysian fluoride guidelines (Malaysian Dental Council, 2009). However, some instructions on the toothpaste packaging did not comply with the national guideline. Certain manufacturers provided misleading messages to the consumer on toothpaste use by using pictorial artwork that implies that the toothpaste used should 'cover the whole length of the bristles'. It is unhelpful to have a disparity between exhortations to use small amounts, while the images depict generously applied toothpaste to the toothbrush (Murray et al., 2003). Evidence reports that the amount of toothpaste used could significantly 
affect total fluoride ingestion (GuhaChowdhury et al., 1996). An excessive amount of fluoride ingestion during critical periods of tooth development may increase the risk of developing dental fluorosis.

Based on the present data, there was a wide range of toothpaste prices for different brands ranging from a low, medium to high cost. In this study, the cost classification for analysis purposes was arbitrary as no reference is available in the literature on this subject of interest. Broadly similar patterns were observed for both fluoridated and non-fluoridated toothpaste. However, some of the non-fluoridated toothpaste were marketed as a high-end product, which contributed to a higher mean price for nonfluoridated toothpaste than fluoridated toothpaste. There is no clear pattern between imported and local products regarding cost. Most of the non-fluoridated toothpastes were marketed as herbal-based or organic products. Similar findings were reported by other authors regarding the toothpastes available in India (Khairnar et al., 2017).

There was a small proportion of the identified toothpastes which contained calcium based abrasives e.g. calcium carbonate $\left(\mathrm{CaCO}_{3}\right), \quad$ dicalcium phosphate dihydrate (DCPD), calcium glycerophosphate (CGP). It is important to note that for toothpaste with sodium fluoride and stannous fluoride, the fluoride compounds are not compatible with calcium-containing abrasives (Benzian et al., 2012). These abrasive agents may deactivate some of the fluoride present. In contrast, for fluoridated toothpastes that use sodium monofluorophosphate [NaMFP $\left.\left(\mathrm{Na}_{2} \mathrm{PO}_{3} \mathrm{~F}\right)\right]$, the $\mathrm{PO}_{3} \mathrm{~F}^{2-}$ components have better compatibility with abrasive agents that contain calcium. This is because in $\mathrm{PO}_{3} \mathrm{~F}^{2-}$, the fluoride is firmly bound to the phosphate and therefore cannot be bound to soluble calcium to form insoluble calcium fluoride. These findings have been confirmed by many laboratory studies (Benzian et al., 2012; Carrera et al., 2012; Vorster et al.,
2018). Therefore, it is an important criterion to be considered to determine total and free fluoride content in the toothpastes available in Malaysia for future research.

The lack of enforcement on guidelines for packaging and labelling of toothpastes in Malaysia may increase the chance for false claims toothpastes. Relevant authorities such as the Ministry of Health and the Malaysian Dental Association should play a fundamental role in monitoring these products. This is not only to ensure the product safety and efficacy, but also to provide a clear guideline and strong enforcement on packaging and labelling of toothpaste in Malaysia. This is crucial to ensure effective delivery of fluoride through toothpaste. In the United States, it is mandatory for manufacturers to declare and accurately label the fluoride content of toothpaste (FDA, 1995).

Results from the present study should be interpreted with some limitations. Firstly, it is not possible to differentiate between genuine and fake products during the data collection period. It is acknowledged that counterfeit products are often identical in terms of labelling and packaging that even experts have difficulty in distinguishing the fake products (Benzian et al., 2012). Thus, it is possible that some of the samples included in the study were counterfeit products. Secondly, the toothpaste samples included in this study were only limited to the selected online and offline markets and there is possibility for some toothpastes to have been missed. Results should not be generalised to other samples of the same brands or the overall toothpaste brand. Thirdly, fluoride content in this study was solely identified based on the labelling on the display box. Further laboratory studies are needed to determine total and free fluoride content in the toothpaste. In addition, clear advice that comply with evidence based recommendations about toothpaste use on the label is highly recommended to benefit the consumers. 


\section{CONCLUSION}

There were many types of toothpastes available in the Malaysian market with a growing number of nonfluoridated toothpaste. Manufacturers' recommendations for toothpaste use varied, with a small proportion following evidence-based recommendations. The cost of toothpaste varied greatly across brands ranging from low $(\mathrm{RM}<1)$, medium (RM1$\mathrm{RM} 4)$ to high $(\mathrm{RM}>4)$ cost. There was a lack of standardisation regarding labelling practices of various toothpaste packaging, which may put consumers and dental health professionals at a disadvantage. Findings from this study may be useful to advocate for standardised guidelines for labelling of toothpaste which will benefit the consumer. The comprehensive list of fluoridated and non-fluoridated toothpastes may be useful for clinicians to provide specific advice based on their patient's needs and for research purposes.

\section{REFERENCES}

Aoun A, Darwiche F, Al Hayek S, Doumit J (2018). The fluoride debate: The pros and cons of fluoridation. Prev Nutr Food Sci, 23(3): 171-180. https://doi.org/10.3746/ pnf.2018.23.3.171

Benzian H, Holmgren C, Buijs M, van Loveren C, van der Weijden F, van Palenstein Helderman W (2012). Total and free available fluoride in toothpastes in Brunei, Cambodia, Laos, the Netherlands and Suriname. Int Dent f, 62(4): 213221. https://doi.org/10.1111/j.1875595X.2012.00116.x

Canadian Paediatric Society (2002). The use of fluoride in infants and children. Paediatr Child Health, 7(8): 569-572. https://doi. org $/ 10.1093 / \mathrm{pch} / 7.8 .569$
Carrera CA, Giacaman RA, Muñoz-Sandoval C, Cury JA (2012). Total and soluble fluoride content in commercial dentifrices in Chile. Acta Odontol Scand, 70(6): 583-588. https://doi.org/10.3109/00016357.2011.64 0287

Food and Drug Administration (FDA) (1995). Anticaries drug products for over-thecounter human use; Final monograph. Fed Reg, 60(194): 52474-52510.

Frencken JE, Sharma P, Stenhouse L, Green D, Laverty D, Dietrich T (2017). Global epidemiology of dental caries and severe periodontitis: A comprehensive review. f Clin Periodontol, 44(Suppl 18): S94-S105. https://doi.org/10.1111/jcpe.12677

Goldman AS, Yee R, Holmgren CJ, Benzian H (2008). Global affordability of fluoride toothpaste. Global Health, 4:7. https://doi. org/10.1186/1744-8603-4-7

Guha-Chowdhury N, Drummond BK, Smillie AC (1996). Total fluoride intake in children aged 3 to 4 years: A longitudinal study. I Dent Res, 75(7): 1451-1457. https://doi.org/10.1177/002203459607500 70401

Gundavarappa KC, Ramachandra SS, Dicksit DD, Gupta VV (2017). The specifications and ingredients found in commercially available toothpastes. Makara $\mathcal{F}$ Health Res, 21(3): 82-86.

Khairnar MR, Dodamani AS, Karibasappa GN, Naik RG, Deshmukh MA (2017). Efficacy of herbal toothpastes on salivary $\mathrm{pH}$ and salivary glucose: A preliminary study. f Ayurveda Integr Med, 8(1): 3-6. https:// doi.org/10.1016/j.jaim.2016.12.004

Malaysian Dental Council (2009). Position Document: Use Of Fluorides In Malaysia. Kuala Lumpur: Malaysian Dental Council. 
Marinho VC, Higgins JP, Sheiham A, Logan S (2003). Fluoride toothpastes for preventing dental caries in children and adolescents. Cochrane Database Syst Rev, (1): CD002278.

Ministry of Health Malaysia (2018). Annual Report 2016: Oral Health Programme, Ministry of Health Malaysia, pp. 28. Retrieved 5 December 2018, from http:// ohd.moh.gov.my/images/pdf/annual_rpt/ annual_rpt16.pdf

Ministry of Health, New Zealand (2009). New Zealand Guidelines Group. Guidelines for the Use of Fluorides. Wellington: Ministry of Health, New Zealand.

Murray JJ, Nunn JH, Steele JG (eds.) (2003). The Prevention of Oral Disease, 4th edn. Oxford: Oxford University Press.

Musa S, Saub R (1998). Toothpastes available in the Malaysian market. Ann Dent Univ Malaya, 5(1): 45-48. https:/doi. org/10.22452/adum.vol5no1.9

O’Mullane DM, Baez RJ, Jones S, Lennon MA, Petersen PE, Rugg-Gunn AJ et al. (2016). Fluoride and oral health. Community Dent Health, 33(2): 69-99. https://doi. org/10.1922/CDH_3707O'Mullane31
Opeodu OI, Gbadebo SO (2017). Factors influencing choice of oral hygiene products by dental patients in a Nigerian Teaching Hospital. Ann Ib Postgrad Med, 15(1): 5156.

Petersen PE, Ogawa H (2016). Prevention of dental caries through the use of fluoride - the WHO approach. Community Dent Health, 33(2): 66-68. https://doi. org/10.1922/CDH_Petersen03

Public Health England (2017). Delivering Better Oral Health: An Evidence-Based Toolkit For Prevention, 3rd edn. London: Public Health England.

Sandier M (1997). The Regulation of Toothpaste. Retrieved 2 December 2018, from http://nrs.harvard.edu/urn-3:HUL. InstRepos:8846762.

Spencer AJ (2006). The use of fluorides in Australia: Guidelines. Aust Dent F, 51(2), 195-199. https://doi. org/10.1111/j.1834-7819.2006.tb00427.x

Vorster L, Naidoo S, Stauf N, Holmgren C, Benzian H (2018). Fluoride content of toothpastes available in South Africa. Community Dent Health, 35(3): 186-192. https://doi.org/10.1922/ CDH_4294Vorster07 


\section{Appendix 1}

The list of adult toothpastes by brands

\begin{tabular}{|c|c|c|c|}
\hline ID & Brand & ID & Brand \\
\hline 1 & Air-Lift Toothpaste & 33 & Jaris Natural Whitening Toothpaste Miswak \\
\hline 2 & Amway Glister Multi-Action Fluoride Toothpaste & 34 & $\begin{array}{l}\text { L'angelica Toothpaste for Sensitive Teeth with } \\
\text { Ginseng and Goji Berry }\end{array}$ \\
\hline 3 & Angola Extra Fresh toothpaste & 35 & Laser 3 Colour Toothpaste \\
\hline 4 & AP24 Whitening Fluoride Toothpaste & 36 & Li-Zey White Boom LED Whitening Toothpaste \\
\hline 5 & Arm \& Hammer Advance White Toothpaste & 37 & Medicam Dental Cream \\
\hline 6 & Atomy Toothpaste & 38 & Melaleuca Whitening Tooth Polish Paste with Fluoride \\
\hline 7 & Bamboo Salt toothpaste & 39 & Morning Kiss Fluoride Toothpaste (Tea Tree Oil) \\
\hline 8 & $\begin{array}{l}\text { Beyond }{ }^{\oplus} \text { Pearl White Whitening Toothpaste } \\
\text { Advanced Formula }\end{array}$ & 40 & Neem Active Toothpaste \\
\hline 9 & BlanCo Fresh \& Natural Fluoride Toothpaste & 41 & Oradex Periodontal Vitamin Toothpaste \\
\hline 10 & Ciptadent Maxi 12 Pus Toothpaste & 42 & Oral 7 Moisturising Toothpaste \\
\hline 11 & Closeup Ever Fresh Toothpaste & 43 & $\begin{array}{l}\text { Oral-B Tooth and Gum Care Fresh Mint Flavor } \\
\text { Toothpaste }\end{array}$ \\
\hline 12 & Colgate Maximum Cavity Protection & 44 & Parodontax Daily Fluoride Toothpaste \\
\hline 13 & Colgate Optic White Toothpaste & 45 & Patanjali Dant Kanti Medicated Oral Gel \\
\hline 14 & Crescent Anti-Cavity Fresh Mint Toothpaste & 46 & Patanjali Dant Kanti Natural Toothpaste \\
\hline 15 & $\begin{array}{l}\text { Crest 3D White Brilliance Fluoride Anticavity } \\
\text { Toothpaste }\end{array}$ & 47 & $\begin{array}{l}\text { Pearlie White Advanced Whitening Fluoride } \\
\text { Toothpaste }\end{array}$ \\
\hline 16 & Curaprox Enzycal 1450 ppm toothpaste & 48 & Pearls Drop Extra Whitening Smokers Toothpaste \\
\hline 17 & Darlie All Shiny White Supreme Toothpaste & 49 & Pepsodent $\odot$ Cavity Fighter Toothpaste \\
\hline 18 & Darlie Double Action Toothpaste & 50 & Pierrot Whitening 2in1 Toothpaste \\
\hline 19 & Dentobac Neem Toothpaste & 51 & Pro Dental B Fresh Mint Toothpaste with Fluoride \\
\hline 20 & DentYucral D Toothpaste & 52 & Pyodontyl Plus F Toothpaste \\
\hline 21 & Dr Rashel Charcoal Whitening Toothpaste & 53 & Rapid White Daily Whitening Toothpaste \\
\hline 22 & Econsave Choice Fluoridated Toothpaste & 54 & $\begin{array}{l}\text { Safi Ubat Gigi Mikro Kalsium Dengan Kayu Sugi \& } \\
\text { Habbatus Sauda }\end{array}$ \\
\hline 23 & Fluocaril Gum Care Toothpaste & 55 & Sayuti Kayu Sugi Toothpaste \\
\hline 24 & Fluoride Toothpaste & 56 & Sensodyne ${ }^{\circ}$ Repair and Protect Toothpaste \\
\hline 25 & Follow Me Extra Cool Toothpaste & 57 & Signal Toothpaste Cavity Fighter \\
\hline 26 & Formula Sparkling White Toothpaste & 58 & Siwak.F Toothpaste \\
\hline 27 & Fresh \& White Triple Cavity Protection Toothpaste & 59 & Sparkle Toothpaste \\
\hline 28 & Genesis ProGum Care & 60 & Sunstar Ora 2 Stain Clear Toothpaste \\
\hline 29 & Giant Cool Mint Toothpaste & 61 & T-Care Sensitive Toothpaste \\
\hline 30 & Gum ${ }^{\circledast}$ Paroex Daily Prevention Toothpaste & 62 & Tesco Choice Freshmint Toothpaste \\
\hline 31 & Himalaya Complete Care Toothpaste & 63 & Tesco Everyday Value Toothpaste \\
\hline 32 & Jaris Natural Mineral Toothpaste - Dead Sea Salt & 64 & TheraBreath Fresh Breath Toothpaste \\
\hline
\end{tabular}


Appendix 1 (Continued)

\begin{tabular}{|c|c|c|c|}
\hline ID & Brand & ID & Brand \\
\hline 65 & Wakamoto Avantbise Toothpaste & 97 & Grants Mild Mint with Aloe Vera Toothpaste \\
\hline 66 & Watsons Freshmint Toothpaste & 98 & GreenZhi ToothGel \\
\hline 67 & White Glo Extra Strength Whitening Toothpaste & 99 & Halagel Miswakgel Toothpaste \\
\hline 68 & Winz Fluoride Toothpaste & 100 & Halagel With Neem and Clove Oil \\
\hline 69 & Xerostom With Saliactive Toothpaste & 101 & HPA Herbal Toothpaste \\
\hline 70 & Yaqeen Betel Leaf and Propolis Toothpaste & $102^{\wedge}$ & K.P. Namboodiri's Ayurvedic Tooth Care Powder \\
\hline 71 & Zact Whitening Toothpaste & 103 & K.P.Namboodiri's Ayurvedic Gel Toothpaste \\
\hline 72 & Alive Natural Propolis Toothpaste & 104 & Leroselle Innovative Natural Toothpaste \\
\hline 73 & All white Smokers' Toothpaste & 105 & Litna Herbal Toothpaste \\
\hline 74 & Amboinense Toothpaste & 106 & LMZ Herbal Gum Specialist Toothpaste \\
\hline 75 & As Shifa Herbal Toothpaste & 107 & $\begin{array}{l}\text { Logodent Daily Care Organic Peppermint } \\
\text { Toothpaste }\end{array}$ \\
\hline 76 & Benfite toothpaste & 108 & $\begin{array}{l}\text { Manuka Honey \& Propolis Toothpaste with } \\
\text { Manuka Oil }\end{array}$ \\
\hline 77 & Bioaqua Herbal Mint Fresh Toothpaste & 109 & Manuka Vantage Toothpaste \\
\hline 78 & Biorepair ${ }^{\oplus}$ Plus Total Protection Toothpaste & 110 & Marine Essence Toothpaste \\
\hline 79 & BMS Organics Aloe Vera Toothpaste & 111 & Marvis Cinnamon Mint Toothpaste \\
\hline $80 \wedge$ & Charcoal Toothpaste Powder & 112 & Mericle 2 Action Whitening Toothpaste \\
\hline 81 & Ciqlo Toothpaste Dr Rodzi Advance Formula & 113 & Miswak Herbal Toothpaste \\
\hline $82^{\wedge}$ & Clobas Tooth Powder & 114 & Mu'min B'Sugi \\
\hline 83 & Coolwhite Toothpaste & 115 & Mu'min Premium Toothpaste - Extra Natural Mint \\
\hline 84 & $\begin{array}{l}\text { Coslys Toothpaste Gel for Sensitive Teeth and } \\
\text { Gums }\end{array}$ & 116 & Najwa Toothpaste \\
\hline 85 & $\begin{array}{l}\text { Dabur Herb'l Natural Toothpaste Neem for Gum } \\
\text { Care }\end{array}$ & 117 & Natura House Herbal Extra Sensitive Toothpaste \\
\hline 86 & Dabur Red Toothpaste & 118 & $\begin{array}{l}\text { Natural Activated Organic Bamboo Charcoal } \\
\text { Toothpaste }\end{array}$ \\
\hline 87 & Dentamate Herbal Toothpaste & 119 & Nazif Toothpaste \\
\hline 88 & Denticare Toothpaste & 120 & $\begin{array}{l}\text { NueVco Extra Virgin Coconut Oil Brightening } \\
\text { Toothpaste }\end{array}$ \\
\hline 89 & Dentiste' Night Time Toothpaste & 121 & Oral Care Whitening Toothpaste \\
\hline 90 & $\begin{array}{l}\text { Dentobac Cool AXN Whitening Herbal Striped } \\
\text { Toothpaste }\end{array}$ & 122 & Orifera Natural Virgin Coconut Oil Toothpaste \\
\hline 91 & Dentobac Gel Natural Herbal Toothpaste & 123 & Oxyfresh Toothpaste \\
\hline 92 & $\begin{array}{l}\text { D dōTERRA On Guard® Natural } \\
\text { WhiteningToothpaste }\end{array}$ & 124 & $\begin{array}{l}\text { Propolise Oral Care Stain Removal Teeth Cleaning } \\
\text { Dental Cream Toothpaste }\end{array}$ \\
\hline 93 & Dr. Neem Herbal Toothpaste & 125 & Pureen Maternity Toothpaste \\
\hline 94 & DXN Ganozhi Toothpaste & 126 & Raiya Go Fresher Non Fluoride Toothpaste \\
\hline 95 & Forever Living Bright Tooth gel & 127 & Rasyan Herbal Clove Toothpaste \\
\hline 96 & $\begin{array}{l}\text { Gano Fresh Toothpaste with Ganoderma Lucidum } \\
\text { Extract }\end{array}$ & 128 & Red Seal Propolis Natural Oral Protection \\
\hline
\end{tabular}




\section{Appendix 1 (Continued)}

ID Brand ID Brand

129 Roselle Toothpaste

130 Roselle Toothpaste Collagen

131 Shabondama Toothpaste

132 Splat Special Whitening Toothpaste Gold

133 Taybah Toothpaste

134 Tinnergy Toothpaste Olive Oil

135 TruCare Nano Silver Toothpaste

136 Turbo Carbon Activated Charcoal Toothpaste
$137 \wedge$ Turbo Carbon Super Polish Teeth Whitening

138 Vibrant Bright Toothpaste

139 Vicco Herbal Toothpaste

140^ Vicco Vajradanti Pure Herbal Tooth Powder

141 Xylin Gamat Toothpaste

142 Xylin Specialist Toothpaste

143 XyliWhite Refreshmint Toothpaste Gel

144 Young Living- Thieves Aroma Bright Toothpaste

Note: ^Powder-based product; ID 1 to 71 is indicated for fluoridated toothpaste; ID 71 to 144 is indicated for non-fluoridated toothpaste. 